\title{
Measurement of the Effect of Monovalent Cations on RNA Hairpin Stability
}

\author{
Jeffrey Vieregg ${ }^{*}$, Wei Cheng, Carlos Bustamante, and Ignacio Tinoco Jr. \\ Contribution from the Department of Physics and Department of Chemistry, University of California, \\ Berkeley and Physical Biosciences Division, Lawrence Berkeley National Laboratory, Berkeley, CA \\ 94720
}

\section{Abstract}

Using optical tweezers, we have measured the effect of monovalent cation concentration and species on the folding free energy of five large (49-124 nt) RNA hairpins, including HIV-1 TAR and molecules approximating A.U and G.C homopolymers. RNA secondary structure thermodynamics are accurately described by a model consisting of nearest-neighbor interactions and additive loop and bulge terms. Melting of small $(<15 \mathrm{bp}$ ) duplexes and hairpins in $1 \mathrm{M} \mathrm{NaCl}$ has been used to determine the parameters of this model, which is now used extensively to predict structure and folding dynamics. Few systematic measurements have been made in other ionic conditions or for larger structures. By applying mechanical force, we measured the work required to fold and unfold single hairpins at room temperature over a range of cation concentrations from 50 to $1000 \mathrm{mM}$. Free energies were then determined using the Crooks Fluctuation Theorem. We observed the following: 1. In most cases, the nearest neighbor model accurately predicted the free energy of folding at $1 \mathrm{M} \mathrm{NaCl}$. 2 . Free energy was proportional to the logarithm of salt concentration. 3. Substituting potassium ions for sodium slightly decreased hairpin stability. The TAR hairpin also misfolded nearly twice as often in $\mathrm{KCl}$, indicating a differential kinetic response. 4. Monovalent cation concentration affects RNA stability in a sequence-dependent manner. G.C helices were unaffected by changing salt concentration, A.U helices were modestly affected, and the hairpin loop was very sensitive. Surprisingly, the UCU bulge of TAR was found to be equally stable in all conditions tested. We also report a new estimate for the elastic parameters of single-stranded RNA.

\section{Introduction}

RNA plays many vital roles in the cell. In addition to carrying information, RNA molecules have been shown to act as catalysts, structural elements, and in regulation of gene expression. For nearly all these functions, folding of linear RNA molecules into specific three-dimensional structures is vital. Prediction of the final structure(s) and folding pathway for an RNA from its sequence is known as the RNA folding problem; it is one of the outstanding problems of biophysical chemistry 1,2 .

The problem is simplified considerably by the observation that RNA folding is largely hierarchical; secondary structure (base paired helices, plus unpaired loops, bulges, and junctions) forms first and is responsible for most of the stability of the fold. One of the most common secondary structures is the hairpin, in which an RNA molecule folds back on itself to form a helical stem capped by an unpaired loop region. Tertiary interactions occur, for the most part, between pre-formed secondary structure elements. In contrast, protein folding thermodynamics is highly context-dependent. Understanding the folding of RNA secondary

E-mail: jvieregg@berkeley.edu. 
structure elements such as hairpins is therefore an important step toward solving the overall problem.

A second simplification was provided by the discovery that RNA secondary structure thermodynamics can be described by a model consisting solely of nearest-neighbor interactions between base pairs plus additive penalties for loops, bulges, and junctions ${ }^{3}$. The parameters of this model have been determined by a large series of measurements at $1 \mathrm{M} \mathrm{NaCl}^{4}$ and are widely used to predict RNA structure 5 and folding dynamics ${ }^{6}$. These measurements were made on small ( $\leq 15 \mathrm{bp}$ ) duplexes or hairpins; larger structures are too stable to easily melt in physiological buffers.

In the cell, monovalent cation concentrations are typically much lower $(\sim 150 \mathrm{mM})$, and $\mathrm{K}^{+}$is the dominant species. As a highly charged polyelectrolyte, RNA is strongly affected by the ionic environment; by screening the phosphates' negative charge, cations make it possible for short range interactions such as hydrogen bonding and base stacking to stabilize compact structures. Theoretical treatments of polyelectrolytes in ionic solution 7,8 commonly treat nucleic acid molecules as line charges that localize a sheath of cations nearby. Since the charge density is greater for folded RNA than when it is unfolded, a number of cations $\Delta \mathrm{n}$ are released to the solution upon unfolding. For monovalent cations, the free energy associated with this increase of entropy is $\mathrm{kT} \Delta \mathrm{n} \ln (\mathrm{I})$, where $\mathrm{k}$ denotes Boltzmann's constant, $\mathrm{T}$ the temperature in Kelvin, and I the ionic strength. This is known as the polyelectrolyte effect. Some models also predict significant length-dependent effects due to the reduction in electric field near the ends of the polyelectrolyte.

The influence of cation concentration on RNA thermodynamics has been measured for both duplexes 9,10 and hairpins ${ }^{11,12}$. While the general trends are consistent, i.e. linear dependence of stability on the logarithm of salt concentration, there appears to be no general formula that encapsulates the data and allows prediction for other molecules. There are far more measurements available for DNA, and several competing formulae that describe the influence of cations on melting thermodynamics $7,13,14$. In particular, there seems to be disagreement in the literature as to whether or not cations stabilize G.C and A.U-rich sequences equally and on the relative importance of end effects.

All the measurements available to date rely on thermal melting of relatively small duplexes and hairpins. As noted above, it is difficult to denature many RNA secondary structures by thermal methods in physiological buffers. Recently, however, it has become possible to apply controlled mechanical force to single nucleic acid molecules using optical tweezers. This makes it possible to characterize the folding of RNA molecules over a wide range of temperatures in nearly any buffer, as first demonstrated by Liphardt et al. in $2001^{15}$. Figure 1 shows a schematic drawing of the optical tweezers instrument used in this study. The RNA molecule is hybridized to two single-stranded DNA handles. The handles are functionalized with biotin and digoxigenin, and bind to streptavidin and anti-digoxigenin coated $\mu \mathrm{m}$-sized polystyrene beads. One bead is fixed to a movable micropipette, and the other is captured in a dual-beam optical trap ${ }^{16}$. The position of the micropipette is controlled with a piezoelectric actuator. Movement of the pipette bead away from the trap stretches the RNA tether; the force is measured directly via the deflection of the trapping laser beams by the trapped bead. This instrument is capable of force and spatial resolutions of $0.1 \mathrm{pN}$ and $1-2 \mathrm{~nm}$ at $100 \mathrm{~Hz}$ bandwidth.

Moving the micropipette at a constant rate while measuring the force yields a characteristic force-extension curve for an RNA molecule, as shown in Figure 2 for the TAR HIV-1 hairpin. As the force is increased, the extension of the molecule increases due to straightening of the handles, which act as entropic springs. At a force of $\sim 19 \mathrm{pN}$, the hairpin unfolds, resulting in a rapid increase in extension and decrease in force. After unfolding, the extension continues 
to increase as the handles and newly unfolded RNA are stretched further. When the pipette motion is reversed, the force decreases and the molecule refolds, in several steps in this case.

Integrating $\mathrm{F} \cdot \mathrm{dx}$ along the curve (light blue) gives the work done stretching the hairpin between the force limits. This work is the sum of three terms (we assume the folded hairpin is rigid):

$$
W=\Delta G_{\text {fold, }, 0 \mathrm{~N}}+W_{\text {stretch }}+W_{\text {diss }}
$$

In addition to the folding free energy at zero force $\Delta \mathrm{G}_{f o l d, 0 p N}$, work $\mathrm{W}_{\text {stretch }}$ is done stretching the handles and unfolded RNA to the final force. If the elastic properties of the handles and RNA are known, this work can be calculated and subtracted from the measured work. As can be seen from the figure, the hairpin unfolds at a higher force than it folded. This hysteresis is a signature of a process taking place away from equilibrium, so additional work $\mathrm{W}_{\text {diss }}$ is dissipated in accordance with the Second Law of Thermodynamics. A recent advance in nonequilibrium statistical physics, the Crooks Fluctuation Theorem $(\mathrm{CFT})^{17}$, makes it possible to recover the reversible work from measurements of an irreversible process such as this. The CFT relates the probabilities of producing a given amount of entropy for a process and its reverse. This yields the following expression relating the work distributions $P_{F, U}(W)$ for folding and unfolding to the free energy $\Delta \mathrm{G}$ :

$$
\frac{P_{U}(W)}{P_{F}(-W)}=e^{W-\Delta G}
$$

The CFT has been used to determine equilibrium work for several systems, including $\mathrm{RNA}^{18}$. This article describes the application of the CFT and optical tweezers to determine how monovalent cation concentration and species affect the stability of RNA secondary structure and to test the accuracy of the nearest neighbor model for larger structures.

\section{Experimental Methods}

\section{RNA Hairpins}

We measured the folding free energy of five RNA hairpins. Their structures are shown in Figure 3 . The structure of HIV-1 TAR has been measured ${ }^{19}$; the rest were calculated using the MFOLD program 5 . For all the hairpins, any predicted alternative structures were significantly less stable than the native fold. We did not observe alternate folding for any hairpin except TAR (Figure 7). TAR's misfolding pattern has been previously characterized ${ }^{20}$ and is easily identifiable from the force-extension curves. Misfolding events lack large folding transitions upon force relaxation and show either no distinct unfolding transition or a "rescue" folding event when the force is subsequently increased. Typical force-extension traces for each hairpin, as well as sample misfolding traces, are included in the supporting information.

\section{Sample Preparation}

Preparation of the TAR and TARdb RNA and handles has been described previously ${ }^{21}$; the 30AU hairpin was prepared in a similar way. DNA templates containing a hairpin sequence and flanking nucleotides (12 nt for TAR, $7 \mathrm{nt}$ for TARdb, no linker for 30AU) were inserted into plasmids (pBR322 for TAR/AUGC/GCAU, pUB/Bsd for TARdb, and pCR2.1 for 30AU) and cloned into E.coli. After culture, a 1200 bp template for RNA transcription and two $\sim 550 \mathrm{bp}$ dsDNA handles were separately amplified by PCR. The handles were then end-labeled with biotin and digoxigenin, respectively. After in vitro transcription by T7 RNA polymerase and purification, the RNA was annealed to the DNA handles. The AUGC and GCAU hairpins required several additional steps of preparation due to their length and the presence of the GC section, as has been described elsewhere ${ }^{22}$. These hairpins were prepared with $39 \mathrm{nt}$ of flanking 
ssRNA. Total handle length was $1112 \mathrm{bp}$ for 30AU and TARdb and $1134 \mathrm{bp}$ for the other molecules. $2.1 \mu \mathrm{m}$ diameter streptavidin-coated polystyrene beads were purchased from Spherotech. Anti-digoxigenin antibody was crosslinked to $2.9 \mu \mathrm{m}$ protein G-coated beads using dimethyl pimelimidate. Only properly-annealed RNA/DNA hybrids are capable of binding to both types of beads.

\section{Optical Tweezers}

Measurements were performed using a dual-beam force measuring optical trap, as described in the introduction. The relative end-to-end extension of the molecule is computed as the difference of the micropipette position and the displacement of the trapped bead from its equilibrium position. The latter was calculated using a harmonic trapping potential with stiffness $9.9 \times 10^{-2} \mathrm{pN} / \mathrm{nm}$. The trap stiffness was measured using a video camera and found to be constant up to at least $50 \mathrm{pN}$. Trap stiffness varied less than $5 \%$ from bead to bead. Data were recorded at $100 \mathrm{~Hz}$.

\section{Measurement Protocol}

All measurements were made at $22 \pm 1^{\circ} \mathrm{C}$ in buffers containing $20 \mathrm{mM}$ Tris (pH 7.5), $4 \mathrm{mM}$ EDTA, $0.05 \% \mathrm{NaN}_{3}$, and either sodium or potassium chloride. Total $\mathrm{Na}^{+}$concentration from the EDTA and azide components was $19 \mathrm{mM}$. Measurements were made at $\mathrm{NaCl}$ concentrations of 50, 100, 200, 400, 700, and $1000 \mathrm{mM}$. TAR and TARdb were also studied at 100, 400, and $1000 \mathrm{mM} \mathrm{KCl}$. Measurements were performed as follows. Inside a microfluidic flow cell, a streptavidin-coated bead was positioned on the micropipette using suction. RNA/DNA constructs were incubated with anti-digoxigenin beads for 15 minutes, a single bead was captured in the optical trap, and tethers were formed between the two beads. Once a stable tether was formed, the micropipette was moved at a constant rate of $100 \mathrm{~nm} / \mathrm{s}$ back and forth between fixed force limits, typically $3-35 \mathrm{pN}$. Measurements for TAR in $1 \mathrm{M}$ $\mathrm{NaCl}$ were repeated at pulling rates of 50 and $200 \mathrm{~nm} / \mathrm{s}$; the free energies obtained from these trials agreed with the $100 \mathrm{~nm} / \mathrm{s}$ value within $0.5 \mathrm{~kJ} / \mathrm{mol}$. For each salt concentration and molecule, measurements were made on several different bead pairs. The results were internally consistent in all cases except for TAR in $100 \mathrm{mM} \mathrm{NaCl}$. We therefore omitted those data from further analysis.

\section{Data Reduction and Analysis}

Mechanical work was computed for each fold/unfold by integrating the force-extension curves as described in the introduction. Integration bounds were selected for each molecule and salt concentration such that all folding/unfolding events were included. To determine the work done stretching the RNA/DNA handles, regions of the F-X curve flanking the integration window (subtracting the extension of the unfolded hairpin from the high force section) were fit using the wormlike chain (WLC) interpolation formula (F: force, X: molecular extension, L: contour length, k: Boltzmann's constant, T: temperature in Kelvin) ${ }^{23}$ :

$$
F=\frac{k T}{4 P}\left(\frac{1}{(1-X / L)^{2}}+\frac{4 X}{L}-1\right)
$$

We fit the persistence length $\mathrm{P}$ as well as an offset in $\mathrm{X}$ individually for each fold and unfold record. We used a value of $0.30 \mathrm{~nm} / \mathrm{bp}$ for the handle contour length, corresponding to an Aform RNA helix ${ }^{24}$. The fit quality was quite good for almost every curve, as exemplified by Figure 2; poor fits were discarded. An extensible WLC model ${ }^{25,26}$, which allows for stretching of the backbone, was also considered but did not significantly change the free energy values (supplementary material). The handle stretch work was then computed by integrating the WLC using the fit values for each fold/unfold and this value was subtracted from the measured work. 
The work required to stretch the unfolded RNA to the upper integration bound was calculated by integrating the $\mathrm{WLC}$ formula with parameters $\mathrm{P}=1.26 \mathrm{~nm}$ and $\mathrm{L}=0.61 \mathrm{~nm} / \mathrm{nt}$, as described in the following section. Subtracting this value yielded the (non-equilibrium) work required to unfold the hairpins alone (Eq. 1); typical distributions are shown in Figure 4. $\Delta \mathrm{G}$ was then determined using the maximum likelihood estimator for the Crooks Fluctuation Theorem derived by Shirts $e t$ al. $^{27}$

\section{Estimation of Uncertainties}

Statistical uncertainties $(1 \sigma)$ for the free energies were computed using the variance expression derived in Ref. 27 , and also by 200 balanced bootstrap resamplings. Both methods gave consistent results, which were less than $2 \%$ of the free energy for all conditions tested. The largest systematic uncertainty comes from the force calibration, which we estimate was accurate to at least $5 \%$ based on comparison of calibrations over time. The "light lever" used to measure micropipette position was calibrated before each experimental session; we estimate no more than a $1 \%$ error from this source. The stiffness of the optical trap was constant to within 5\%, but these variations are largely compensated by the handle fitting procedure; varying the stiffness value over this range changed the free energy by $\sim 1 \%$. A final source of error is the subtraction of the stretch work; exploration of the WLC parameter space showed that this also affected the free energy values at a $1 \%$ level. The uncertainties do not appear to be correlated, leading to a total systematic uncertainty estimate of 5.3\%.

\section{Results}

\section{Elastic Properties of Single-stranded RNA}

In order to determine the folding free energy at zero force, the work required to stretch the handles and unfolded RNA to the final force must be subtracted from the measured work values (Eq. 1). The handle work is available from the measured force-extension curve but the work done on the unfolded RNA must be calculated. A two parameter WLC model (3) is often used to describe nucleic acid elasticity. Liphardt et al. found a persistence length of $1.0 \mathrm{~nm}$ for singlestrand RNA, assuming a contour length of $0.59 \mathrm{~nm} / \mathrm{nt}$ (C3'-endo sugar pucker) ${ }^{15}$. This persistence length was calculated from the change in extension of the P5ab hairpin at its equilibrium force in a buffer containing $250 \mathrm{mM} \mathrm{NaCl}$ and $10 \mathrm{mM} \mathrm{MgCl}_{2}$. Given the larger range of forces in these measurements and the possibility of salt dependence, we decided to measure the elastic properties in our experimental conditions. We computed the mean force and extension change at each salt concentration for two transitions: unfolding of TAR and folding of the GC section of AUGC. The other transitions were visibly not two-state or displayed rapid hopping (see supplementary material) that made it difficult to accurately measure $\Delta \mathrm{X}$. We also included constant-force hopping data for P5ab (in $250 \mathrm{mM} \mathrm{NaCl}, 1 \mathrm{mM}$ EDTA) from Wen et al. ${ }^{28}$ in the analysis. The data are plotted in Figure 5, along with predictions from the WLC model for various values of the parameters. We obtained the best fit (red line; supplementary material) with a persistence length of $1.26 \pm 0.15 \mathrm{~nm}$ and contour length of $0.61 \pm 0.01 \mathrm{~nm}$, both of which are larger than the literature value (black line). This may be due to the effect of magnesium, as Liphardt et al. report a larger extension/nt when $\mathrm{Mg}^{2+}$ is replaced by EDTA. Within the uncertainty of our measurements, we observed no dependence of the elastic properties on monovalent cation concentration. Adding an enthalpic stretch term to the WLC model did not improve the fit or appreciably alter the single-strand stretching free energy, in agreement with an earlier measurement ${ }^{29}$.

\section{Effect of Cation Species}

To determine whether sodium and potassium affect RNA hairpins differently, we examined the folding of two hairpins, TAR and TARdb. The TAR hairpin is located at the $5^{\prime}$ terminus of the HIV-1 genome and plays vital roles in the viral life cycle ${ }^{30}$. A three nucleotide bulge 
(Figure 3) is bound by the Tat protein; this is essential for viral transcription. As a result, TAR has been studied extensively by both bulk and single-molecule methods. TARdb is a mutant that lacks the $3 \mathrm{nt}$ bulge. Both hairpins are quite stable (predicted melting temperatures of 88.1 and $96.7^{\circ} \mathrm{C}$ in $1 \mathrm{M} \mathrm{NaCl}^{4}$ ), which has prevented measurement of their thermodynamics by thermal melting. Figure 6 shows folding free energy vs. salt concentration for both hairpins (larger numbers $=$ more stable), as well as the values predicted by the nearest-neighbor model (MFOLD 2.3 at $\left.22{ }^{\circ} \mathrm{C}\right)^{5}$. All the molecules studied show a linear dependence of the folding free energy on the logarithm of the salt concentration, as expected. The free energy we observed for TAR in $100 \mathrm{mM} \mathrm{KCl}(135.7 \pm 7.2 \mathrm{~kJ} / \mathrm{mol})$ is significantly larger than that derived from an earlier kinetic study ${ }^{21}$ that assumed two-state folding with a force-independent transition state distance.

TAR has been shown to occasionally undergo folding transitions with a shorter end-to-end extension change (fewer base pairs formed) when folding occurs away from equilibrium. ${ }^{20}$ This has been suggested to reflect formation of non-native structures, i.e. misfolding. In our experimental conditions, these transitions occurred nearly twice as frequently in $\mathrm{KCl}$ as in $\mathrm{NaCl}$ (Figure 7). The misfolding probability appears to be independent of salt concentration. None of the other moecules we studied showed signs of alternative folding.

\section{Sequence Dependence}

In order to determine whether cations affect RNA secondary structure in a sequence-dependent manner, we measured the folding free energy of three hairpins that approximate A.U and G.C homopolymers. The free energy of the 30AU hairpin (Figure 3) is shown in Figure 8. This hairpin is nearly twice as stable at $1 \mathrm{M} \mathrm{NaCl}$ than it is at $50 \mathrm{mM}$, a much larger difference than for any of the other molecules.

The AUGC hairpin contains the A.U section of $30 \mathrm{AU}$, but $30 \mathrm{G} \cdot \mathrm{C}$ base pairs are inserted before the terminal tetraloop. As force is increased, this molecule unfolds in two steps: the A.U helix unfolds reversibly at $12-15 \mathrm{pN}$, and the G.C helix and loop unfold between 25 and $29 \mathrm{pN}$ with noticeable hysteresis. Due to the separation in force, the free energies can be measured independently and are plotted in Figure 9. Both transitions show a modest dependence on $[\mathrm{NaCl}]$ compared to the $30 \mathrm{AU}$ hairpin.

Finally, we measured the stability of the GCAU hairpin, which contains the same A.U and G.C sections arranged in the opposite order, with the more stable G.C pairs at the open end of the hairpin loop. As a result of this arrangement, GCAU displays substantial hysteresis when folding. Unfolding requires a force of $23-28 \mathrm{pN}$ while folding cannot occur until the force drops to $\sim 10 \mathrm{pN}$. This results in a large amount of dissipated work, illustrated by the distance between the work peaks in Figure 4C. Despite this, the folding and unfolding work distributions are relatively narrow and no overlap is seen. At first glance, this is surprising; one expects the work distributions to become wider as the reaction occurs farther from equilibrium ${ }^{18,31}$.

Examination of the force-extension curves for the 30AU and AUGC hairpins reveals a possible explanation, however. Although the overall folding/unfolding reaction takes place far from equilibrium, the rate-limiting steps (folding of A.U and unfolding of G.C base pairs) actually occur near their equilibrium forces, leading to narrow force distributions and thus relatively narrow work distributions. If $\mathrm{A}, \mathrm{B}$, and $\mathrm{C}$ represent the fully-folded, $\mathrm{A} \cdot \mathrm{U}$ region folded, and unfolded states respectively, the reaction kinetics can be schematically written as follows:

$$
\begin{aligned}
& \text { Unfolding: } A \leftrightarrow B \rightarrow C \\
& \text { Folding: } C \leftrightarrow B \rightarrow A
\end{aligned}
$$

The folding free energy for GCAU is shown in Figure 10. 


\section{Discussion}

\section{Accuracy of the Nearest-Neighbor Model}

As discussed in the introduction, a nearest-neighbor model is widely used to predict RNA secondary structure thermodynamics, structure, and folding dynamics. By applying force, we were able to test the model's ability to predict the stability of hairpins that are considerably larger and more stable than those used to derive its parameters. Table 1 shows the predicted and observed Gibbs free energy for all six folding transitions at $1 \mathrm{M} \mathrm{NaCl}$. In each case, we found the hairpins to be more stable than the model predicted. However, the difference was less than or equal to 1.5 standard deviations for all the hairpins except TAR, which is not very significant given that the free energy predictions are subject to uncertainty as well ${ }^{4}$. Considering the large temperature difference between typical melting experiments and this measurement, it is somewhat remarkable that a model that assumes temperature-independent specific heats is as accurate as it appears to be. Another test of the nearest-neighbor model comes from the two longest hairpins. At all salt concentrations, $\Delta \mathrm{G}$ for GCAU is equal (within measurement error) to the sum of the free energies of the two steps required to unfold the AUGC hairpin.

The TAR hairpin is $19 \mathrm{~kJ} / \mathrm{mol}(2 \sigma)$ more stable in $1 \mathrm{M} \mathrm{NaCl}$ than predicted, while the predicted stability of TARdb agrees with measurement. In the framework of the nearest neighbor model, this suggests that the UCU bulge of TAR is more stable than predicted (Figure 12). As this bulge is known to be biologically functional, this is not too surprising. Indeed, genetic analysis suggests that one of the bulge nucleotides forms a base triple with the neighboring base pair 32 .

\section{Effect of Cation Species}

Both TAR and TARdb are somewhat more stable (10-15 kJ/mol) in $\mathrm{NaCl}$ than in $\mathrm{KCl}$, particularly at higher concentrations. $\mathrm{Na}^{+}$and $\mathrm{K}^{+}$ions differ in size (2.0 vs. $\left.2.7 \AA\right)$ and also in their hydration interactions ${ }^{33}$. The difference in folding free energy of the RNA could be due to either differential binding of the cations to specific sites or a more general difference in stabilization ability between the cations. No monovalent cation binding site has been reported for TAR, but the terminal loop is known to be partially structured ${ }^{34}$. In addition to this thermodynamic difference, we also found that sodium and potassium influence the folding kinetics of TAR differently; misfolding occurred more frequently in $\mathrm{KCl}$. Misfolding is thought to occur as a result of formation of non-native terminal loops ${ }^{20}$, which is consistent with preferential binding of $\mathrm{Na}+$ ions by a site in the native 6-loop common to TAR and TARdb. More precise measurements on more molecules are required to answer this question.

\section{Sequence Dependence}

By comparing the free energies of the $30 \mathrm{AU}$ and AUGC transitions, we were able to determine how $\mathrm{NaCl}$ concentration affects different sequences and structures of RNA hairpins. Figure 11 shows the calculated average free energy per base pair for A.U and G.C helices, and the calculated free energies of a $4 \mathrm{nt}$ terminal loop and the UCU bulge of TAR are plotted in Figure 12. The A.U value was obtained directly from the low force transition of AUGC, and subtraction of $\Delta \mathrm{G}$ for that transition from the 30AU free energy yielded the stability of the tetraloop. The average G.C free energy was then calculated from the difference in free energy between the AUGC high force transition and the tetraloop. $\triangle \mathrm{G}$ for the UCU bulge was calculated from the difference between TAR and TARdb. All of these calculations are made within the nearest-neighbor framework, in which the free energies of various elements are purely additive. 
The average free energy of G.C base pairs is (within experimental uncertainty) a constant 14.3 $\pm 0.5 \mathrm{~kJ} / \mathrm{mol}$ over the salt concentrations studied. The stability of A.U base pairs is proportional to $\log [\mathrm{NaCl}]$ as follows: $\Delta \mathrm{G}_{\mathrm{A} \cdot \mathrm{U}}=3.63 \pm 0.58+(0.60 \pm 0.24) \log [\mathrm{NaCl}(\mathrm{mM})]$. Both values are in good agreement with the nearest-neighbor model at $1 \mathrm{M} \mathrm{NaCl}$. The free energy of the tetraloop is much more sensitive to cation concentration: $\Delta \mathrm{G}_{\text {loop }}=-111 \pm 27.4+(31.5 \pm 11.7)$ $\log [\mathrm{NaCl}(\mathrm{mM})]$. The loop is more than three times less stable in $50 \mathrm{mM} \mathrm{NaCl}$ than it is in 1M. From Figure 12, it appears that the tetraloop free energy could also be fit by a function that saturates at higher $\log [\mathrm{NaCl}]$, but we are not aware of a physical model that predicts this. In contrast to the loop, the UCU bulge of TAR appears equally unstable $(-10.8 \mathrm{~kJ} / \mathrm{mol}$ avg. $\Delta \mathrm{G})$ at all concentrations of both sodium and potassium chloride. This may not be representative of other bulges, however, for the reasons discussed earlier. The decrease in stability of the hairpins with salt concentration is thus due primarily to the terminal loop rather than the helices. These results, if general, suggest that the lowest free energy structures predicted by the nearest-neighbor model at $1 \mathrm{M} \mathrm{NaCl}$ may not be the most stable folds at lower salt concentrations.

Even without a full set of nearest-neighbor parameters, it is still possible to predict the salt dependence of hairpin stability based on nucleotide composition alone. Figure 13 shows the predicted folding free energy of TARdb along with the measured values. The calculation makes several assumptions. First, salt is assumed to destabilize the $6 \mathrm{nt}$ loop of TARdb and the tetraloop identically. Second, the stability of the two G.U wobble pairs is calculated as the average of the two types of Watson-Crick pairs. Finally, the $1 \mathrm{nt}$ bulge of TARdb is assumed to be unaffected by cation concentration. While the prediction consistently overestimates the folding free energy, the actual values do agree within the calculated uncertainty at all concentrations. Measurement of nearest-neighbor parameters in these conditions would greatly increase the accuracy of free energy calculations.

\section{Conclusions}

We have measured the free energy of folding for five RNA hairpins in $\mathrm{NaCl}$ and $\mathrm{KCl}$ concentrations from 50 to $1000 \mathrm{mM}$ using optical tweezers. By applying mechanical force, we were able to repeatedly fold and unfold molecules that are too stable to study easily with bulk thermal melting. We found that a nearest-neighbor model accurately predicted the free energies at $1 \mathrm{M} \mathrm{NaCl}$ for all the molecules except the TAR hairpin from HIV-1. TAR and a related mutant were slightly less stable when potassium was substituted for sodium and TAR misfolded nearly twice as frequently in $\mathrm{KCl}$. This may be due to preferential stabilization of the hairpin loop, which is known to be structured. We also studied three hairpins that approximate A.U and G.C homopolymers and found that cation concentration affects RNA stability in a sequence-dependent manner. Base-paired helices were minimally sensitive to salt (G.C base pairs were unaffected, A.U stability decreased slightly with $[\mathrm{NaCl}])$, whereas the hairpin loop was strongly destabilized at lower salt concentrations. Surprisingly, the $3 \mathrm{nt}$ bulge of TAR was equally stable in all conditions. These results, if general, suggest that the most stable secondary structure of RNA molecules may vary depending on the monovalent cation concentration. From these measurements, we were able to predict the salt dependence of the TARdb hairpin folding free energy with reasonable accuracy. Further measurements to derive nearest-neighbor parameters would be very useful for understanding RNA folding in physiological buffer conditions.

\section{Supplementary Material}

Refer to Web version on PubMed Central for supplementary material. 


\section{Acknowledgements}

We are grateful to Dr. Pan Li for providing the TAR and TARdb clones as well as much helpful discussion. We thank Dr. Jin-Der Wen for making the force-extension data of P5ab available and for reviewing the manuscript. We also thank Dr. Andrew Greytak for critical reading of the manuscript. J.V. was supported by the University of California Systemwide Biotechnology Research \& Education Program Graduate Research and Education in Adaptive Biotechnology Training Program, grant \#2005-249. Additional support was provided by NIH grants GM-10840 (I.T.) and GM-32543 (C.B.).

\section{References}

1. Tinoco I, Bustamante C. J Mol Biol 1999;293:271-281. [PubMed: 10550208]

2. Herschlag D. J Biol Chem 1995;270:20871-20874. [PubMed: 7545662]

3. Tinoco I, Uhlenbeck OC, Levine MD. Nature 1971;230:362-367. [PubMed: 4927725]

4. Mathews DH, Sabina J, Zuker M, Turner DH. J Mol Biol 1999;288:911-940. [PubMed: 10329189]

5. Zuker M. Nucleic Acids Research 2003;31:3406-3415. [PubMed: 12824337]

6. Cocco S, Marko JF, Monasson R. Euro Phys J E 2003;10:153-162.

7. Rouzina I, Bloomfield VA. Biophys Journal 1999;77:3242-3251.

8. Anderson CF, Record MT. Annu Rev Phys Chem 1995;46:657-700. [PubMed: 7495482]

9. Krakauer H, Sturtevant JM. Biopolymers 1968;6:491-512. [PubMed: 5644782]

10. Mikulecky PJ, Feig AL. Biopolymers 2006;82:38-58. [PubMed: 16429398]

11. Williams DJ, Hall KB. Biochemistry 1996;35:14665-14670. [PubMed: 8931566]

12. Soto AM, Misra V, Draper DE. Biochemistry 2007;46:2973-2983. [PubMed: 17315982]

13. SantaLucia J. PNAS 1998;95:1460-1465. [PubMed: 9465037]

14. Owczarzy R, You Y, Moreira BG, Manthey JA, Huang L, Behlke MA, Walder JA. Biochemistry 2004;43:3537-3554. [PubMed: 15035624]

15. Liphardt J, Onoa B, Smith SB, Tinoco I, Bustamante C. Science 2001;292:733-737. [PubMed: 11326101]

16. Smith SB, Cui Y, Bustamante C. Methods Enzymology 2003;361:134-162.

17. Crooks GE. Phys Rev E 1999;60:2721-2726.

18. Collin D, Ritort F, Jarzynski C, Smith SB, Tinoco I, Bustamante C. Nature 2005;437:231-234. [PubMed: 16148928]

19. Muesing MA, Smith DH, Capon DJ. Cell 1987;48:691-701. [PubMed: 3643816]

20. Li PTX, Bustamante C, Tinoco I. PNAS 2007;104:7039-7044. [PubMed: 17438300]

21. Li PTX, Collin D, Smith SB, Bustamante C, Tinoco I. Biophys Journal 2006;90:250-260.

22. Cheng W, Dumont S, Tinoco I, Bustamante C. PNAS 2007;104:13954-13959. [PubMed: 17709749]

23. Bustamante C, Marko J, Siggia E, Smith S. Science 1994;265:1599-1600. [PubMed: 8079175]

24. Rich A. Nature Struct Bio 2003;10:247-249. [PubMed: 12660721]

25. Odjik T. Macromolecules 1995;28:7016-7018.

26. Wang MD, Yin H, Landick R, Gelles J, Block SM. Biophys J 1997;72:1335-1346. [PubMed: 9138579]

27. Shirts MR, Bair E, Hooker G, Pande VS. Phys Rev Letters 2003;91:140601.

28. Wen JD, Manosas M, Li PTX, Smith SB, Bustamante C, Ritort F, Tinoco I. Biophys Journal 2007;92:2996-3009.

29. Seol Y, Skinner GM, Visscher K. Phys Rev Let 2004;93:118102. [PubMed: 15447383]

30. Das AT, Klaver B, Berkhout B. J Virol 1998;72:9217-9223. [PubMed: 9765469]

31. Gore J, Ritort F, Bustamante C. PNAS 2003;100:12564-12569. [PubMed: 14528008]

32. Huthoff H, Girard F, Wijmenga SS, Berkhout B. RNA 2004;10:412-423. [PubMed: 14970387]

33. Carrillo-Tripp M, Saint-Martin H, Ortega-Blake I. J Chem Phys 2003;118:7062-7073.

34. Kulinski T, Olejiniczak M, Huthoff H, Bielecki L, Pachulska-Wieczorek K, Das AT, Berkhout B, Adamiak RW. J Biol Chem 2003;278:38892-38901. [PubMed: 12882959] 
Counter-propagating lasers, $\lambda=830 \mathrm{~nm}$

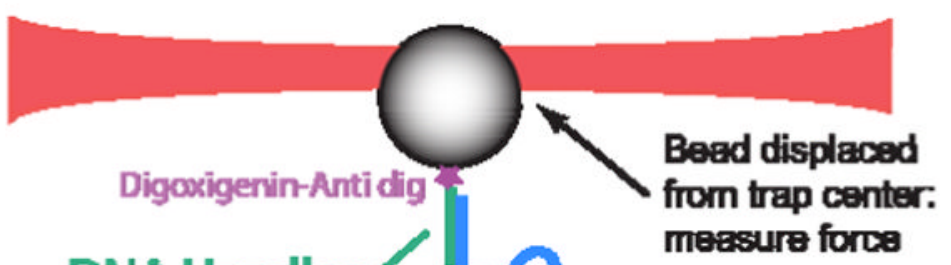

Figure 1.

Experimental Setup (not to scale) 


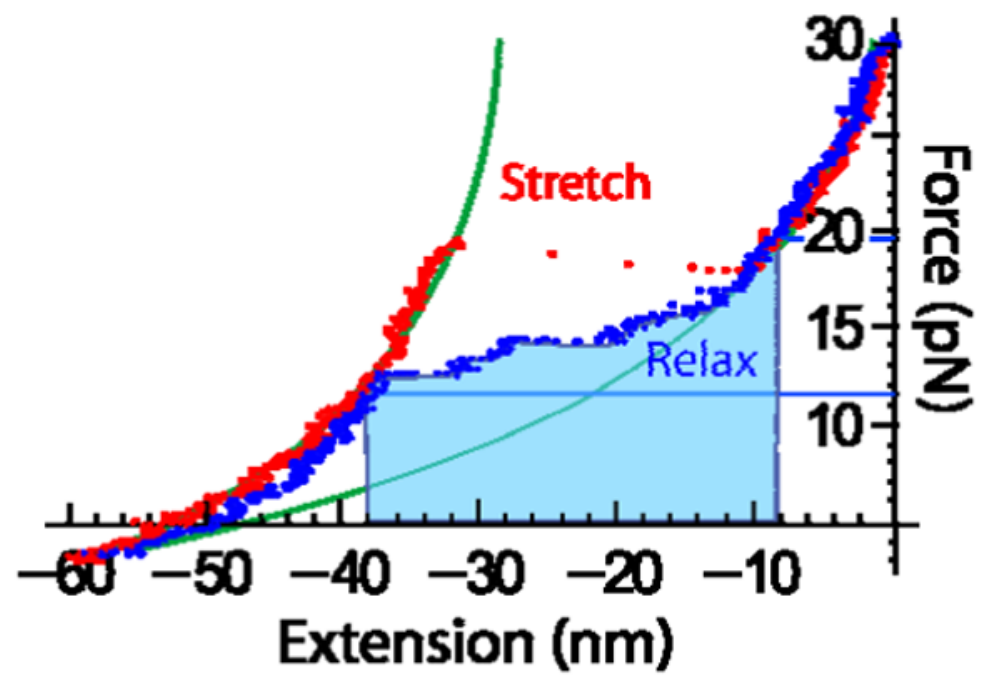

Figure 2.

Typical Hairpin Force-Extension Record (TAR hairpin, 1M NaCl). Abscissa is end-to-end extension of the molecule (hairpin and handles) relative to its value at the peak force. Data (dots) from increasing force are in red and decreasing force are in blue; WLC fits (Eq. 3) are in green for folded, unfolded hairpin. Light blue area shows work integration during decreasing force (folding) trajectory. 
A HIV-1 TAR Halmin

B TARdb Hairpin

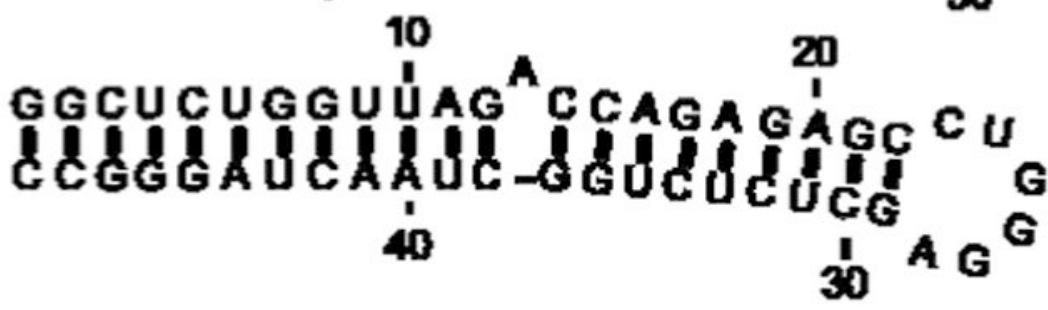

\section{30AU Halrpin}

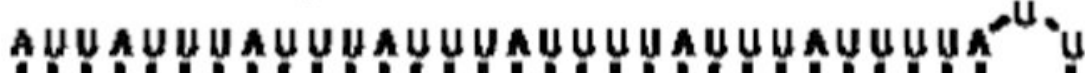

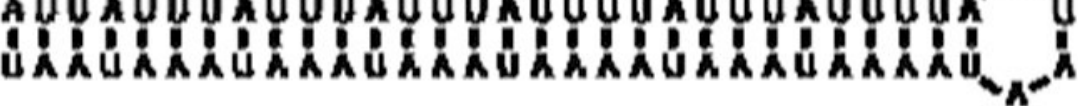

D AUGC Hairpin

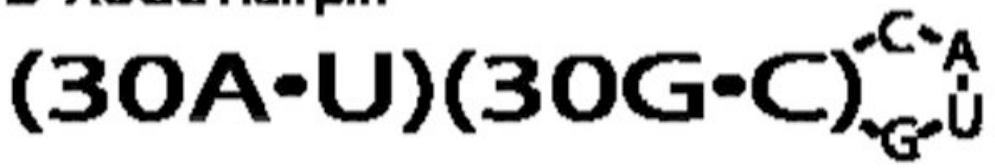

E GCAU Hairpin

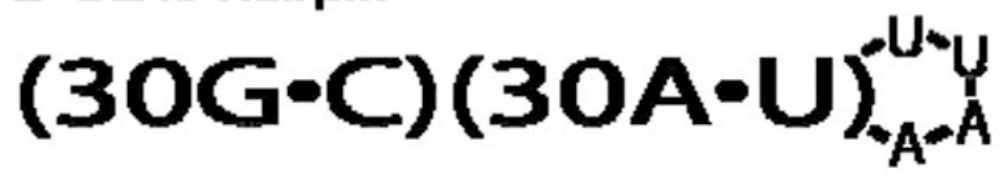

Figure 3.

Hairpin structures 

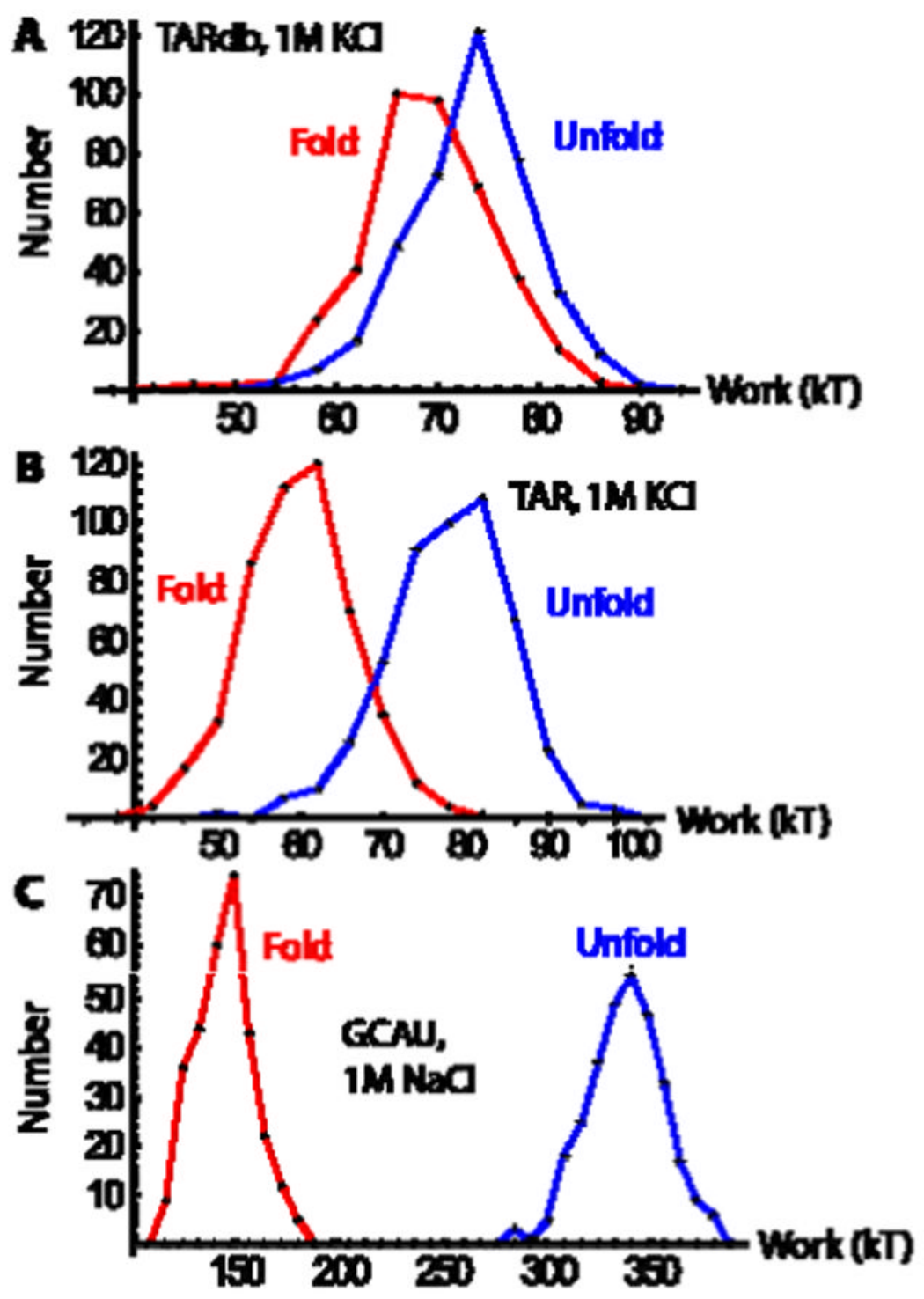

Figure 4.

Typical Work Distributions for (A) TARdb, (B) TAR, (C) GCAU Hairpins. Data binned in 3 $\mathrm{kT}$ intervals for display only. Irreversibility increases from top to bottom. 


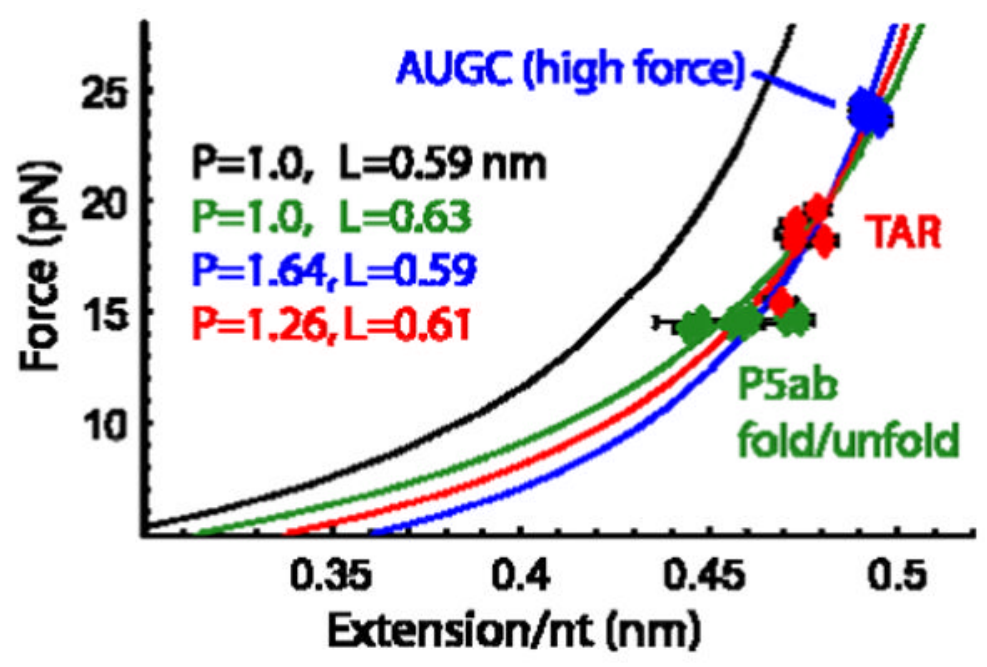

Figure 5.

Force-extension data for three transitions and WLC fits. Extension/nt calculated assuming 2 nm diameter for the folded helix. Error bars are s.e.m. 

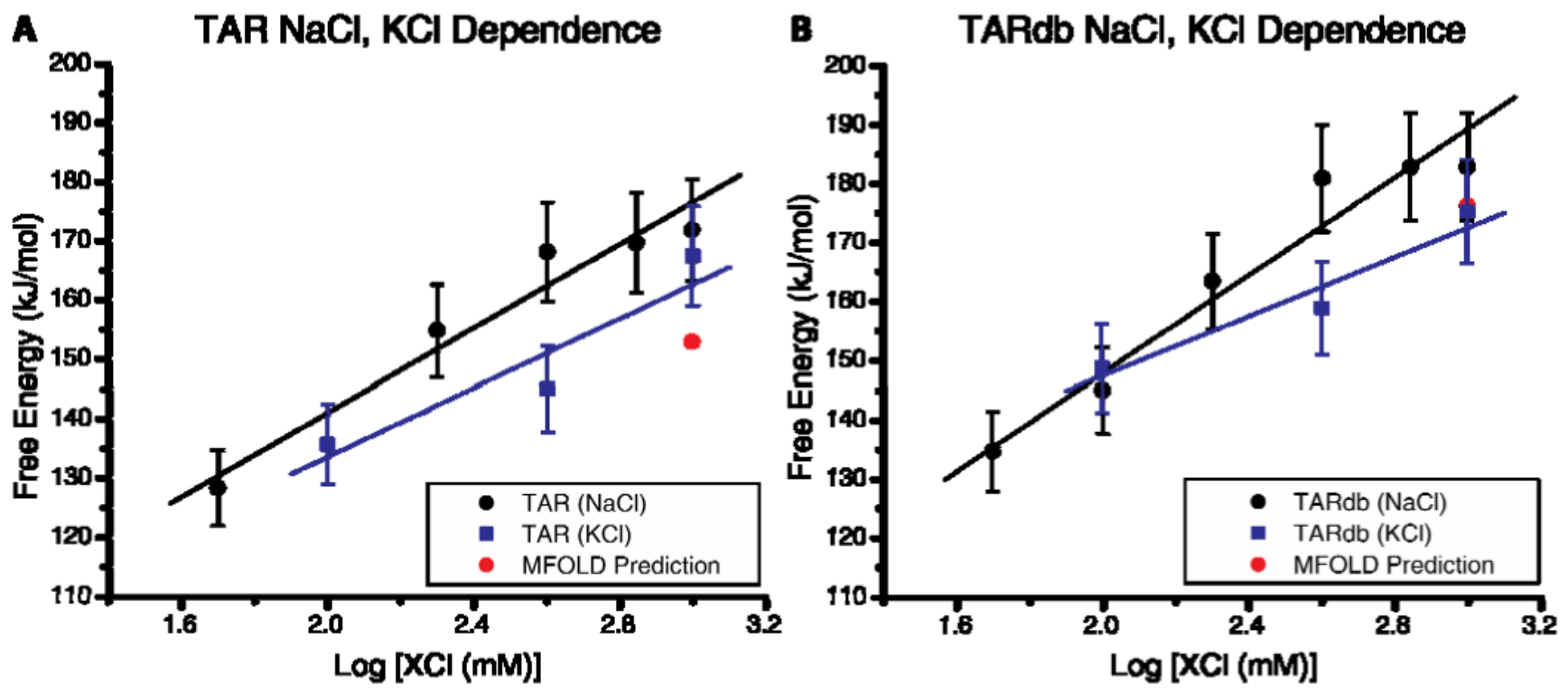

Figure 6.

Free Energy vs. Salt Concentration for TAR (A) and TARdb (B) Hairpins 


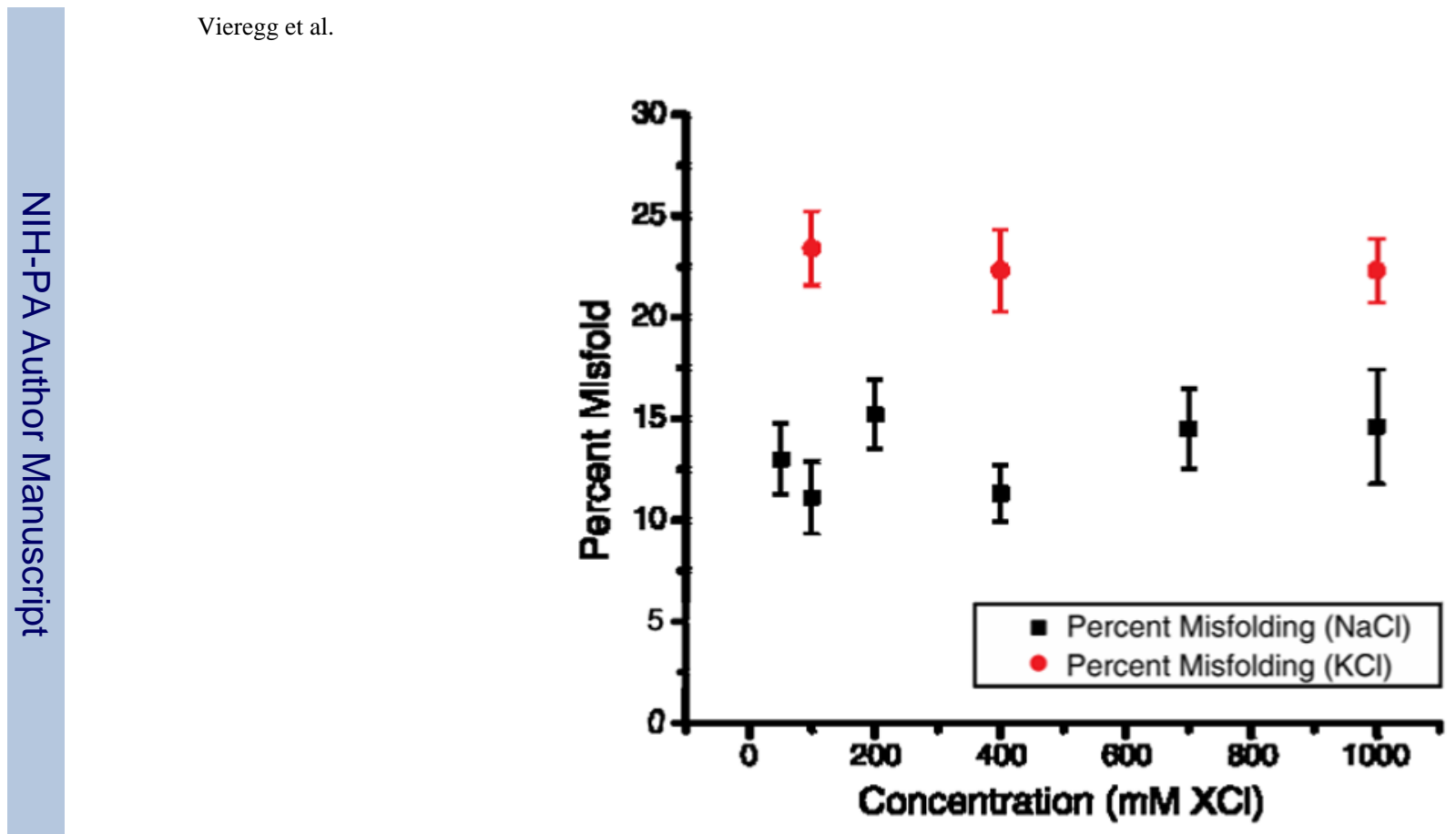

Figure 7.

TAR Misfolding Probability in $\mathrm{NaCl}, \mathrm{KCl}$ 


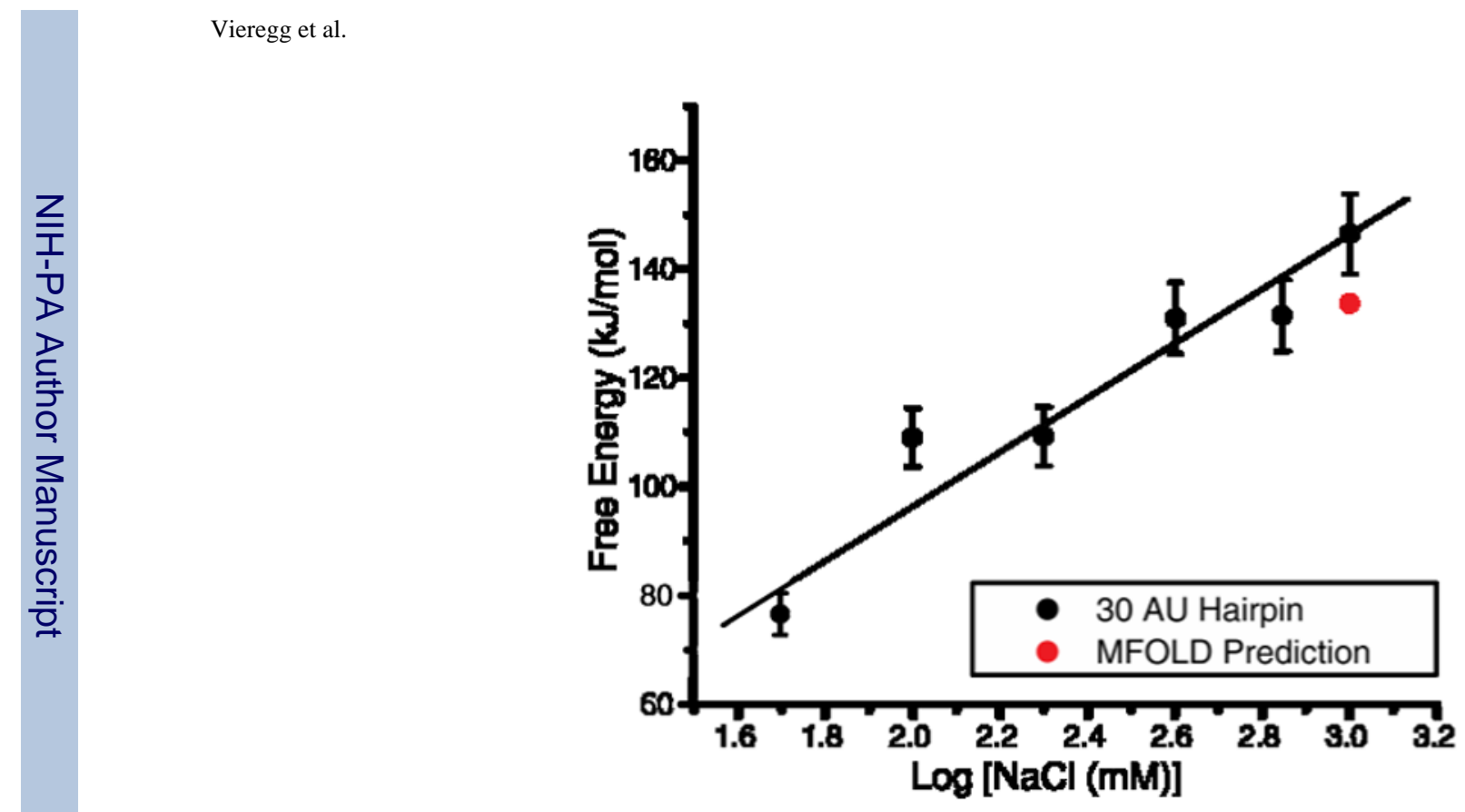

Figure 8.

Free Energy of 30AU hairpin vs. [ $\mathrm{NaCl}]$ 


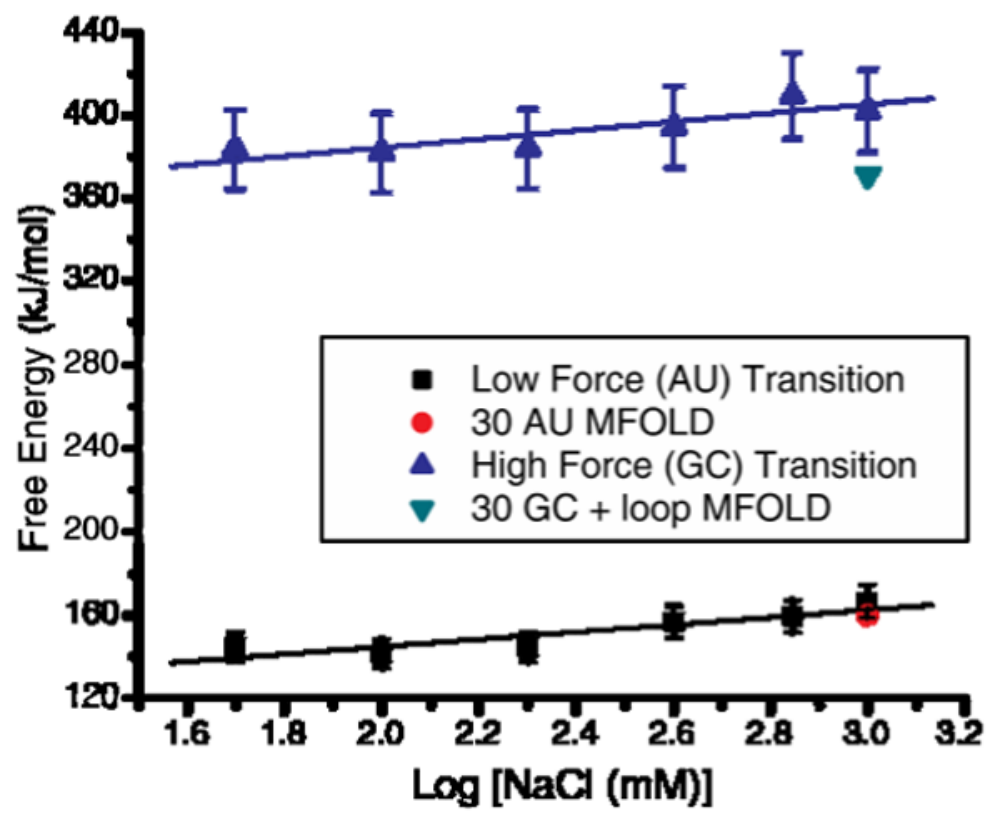

Figure 9.

Free Energy of AUGC Hairpin vs. [NaCl] 


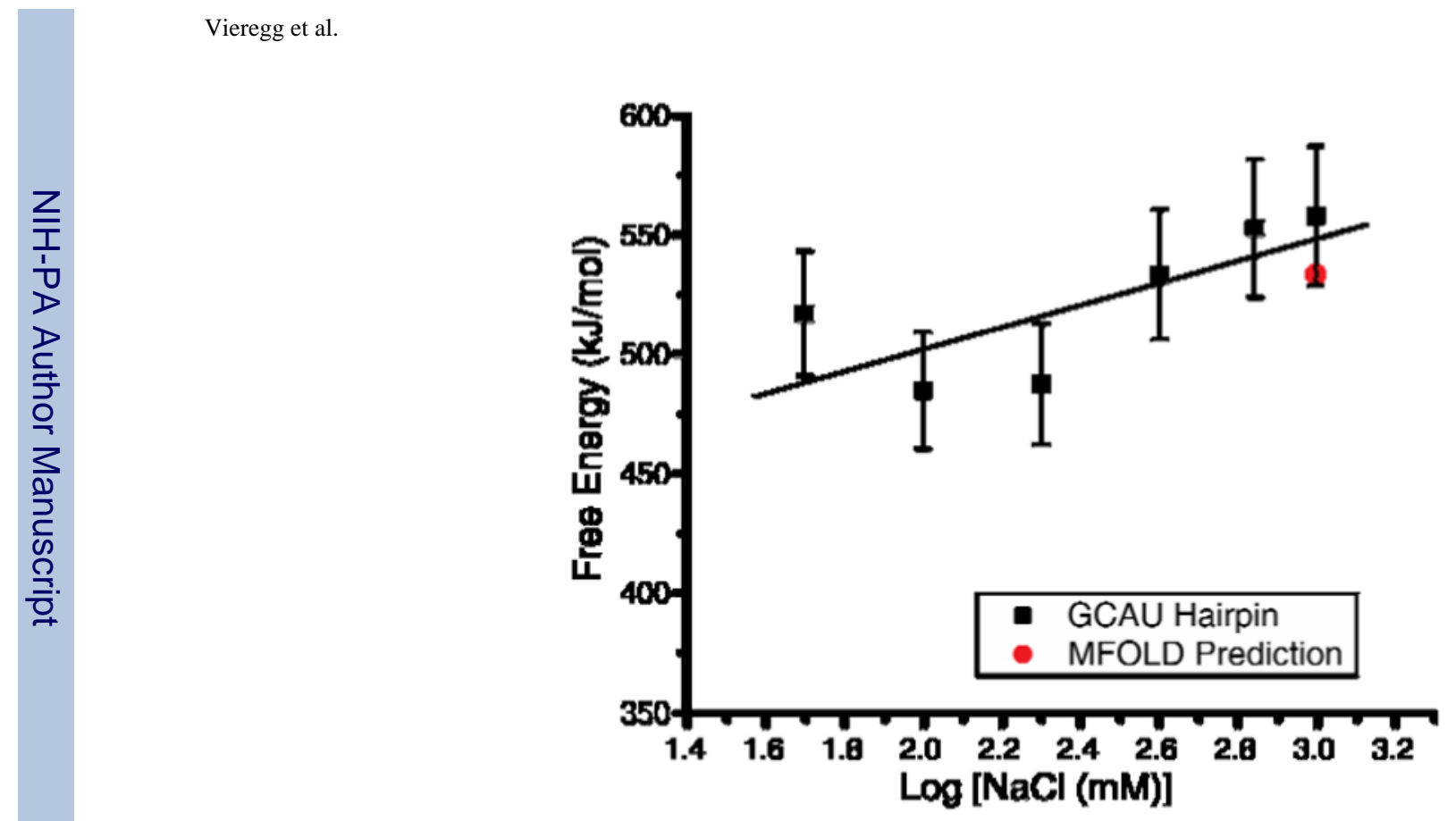

Figure 10.

Free Energy for GCAU Hairpin vs. [NaCl] 


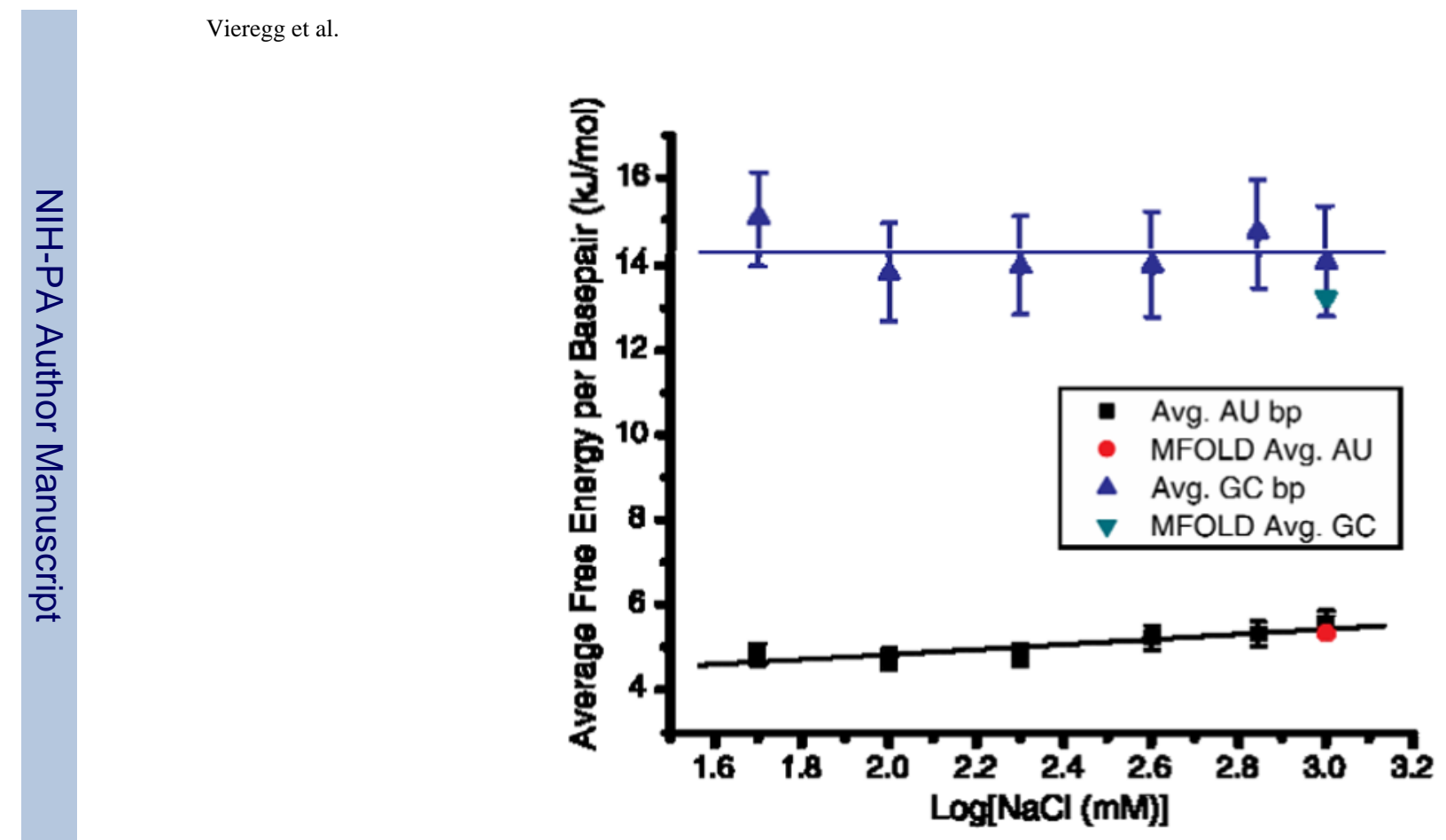

Figure 11.

Average Free Energy of AU, GC Helices 


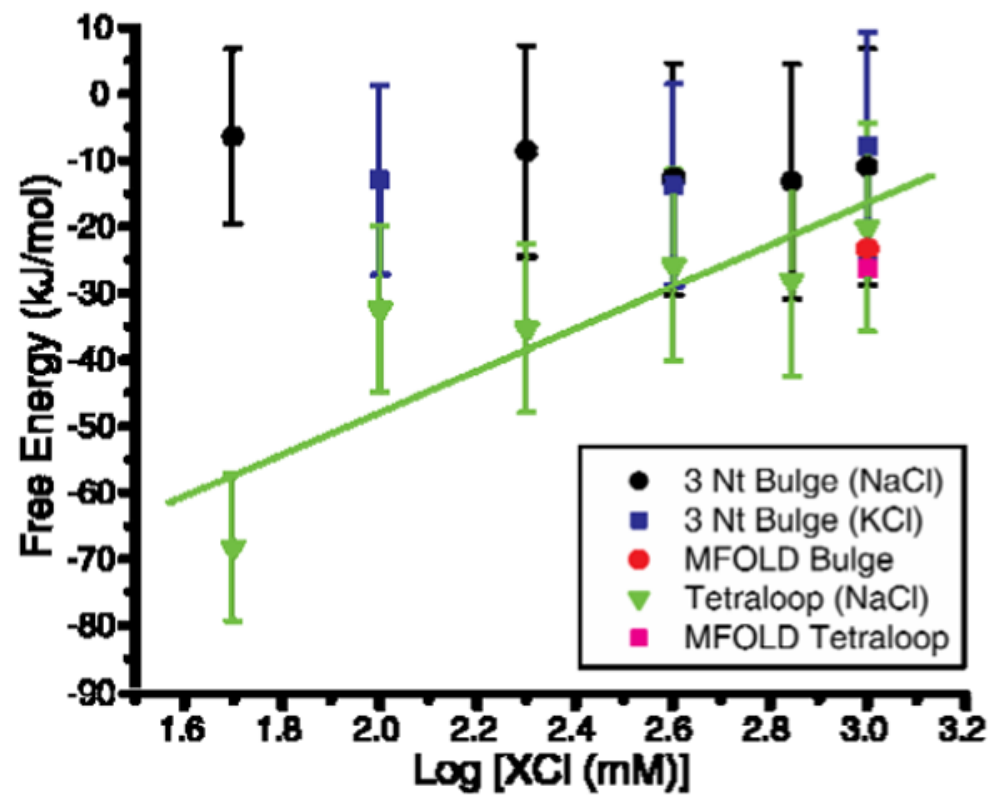

Figure 12.

Loop and Bulge Stability 


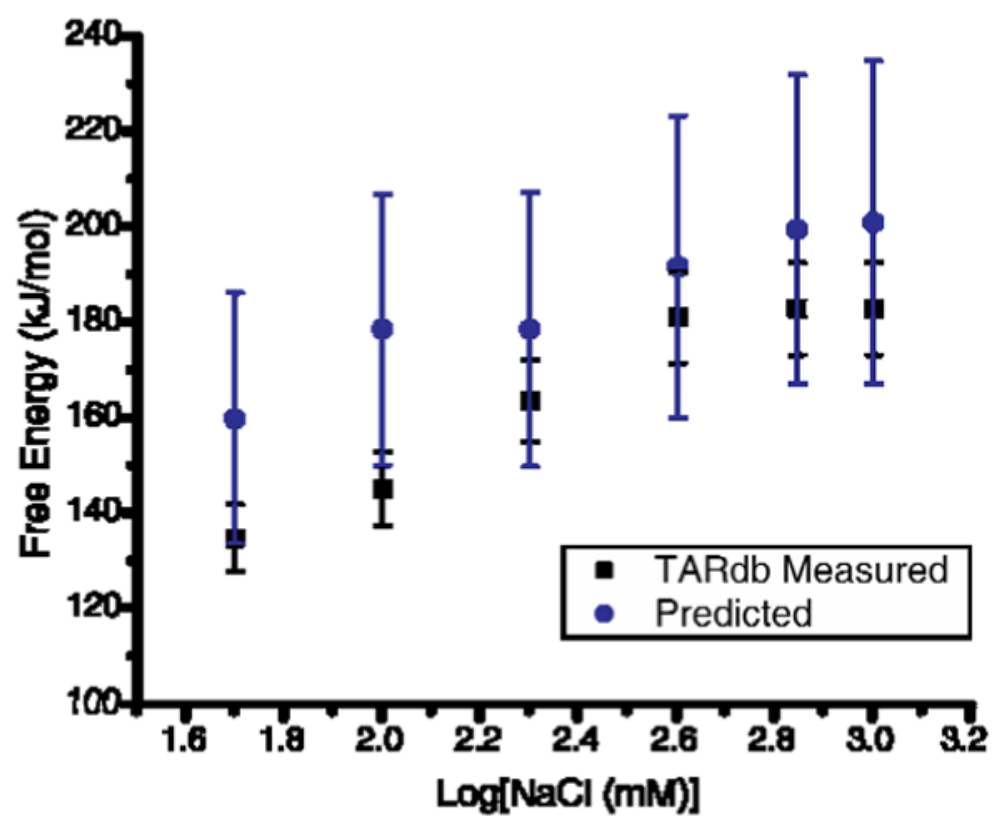

Figure 13.

Predicting TARdb Folding Free Energy 
Table 1

Predicted vs. Measured Folding Free Energy at $1 \mathrm{M} \mathrm{NaCl}$

\begin{tabular}{|c|c|c|c|c|}
\hline Hairpin & $\begin{array}{c}\text { Length } \\
(\mathbf{n t})\end{array}$ & $\begin{array}{c}\mathbf{G} \cdot \mathbf{C} \\
(\mathbf{\%})\end{array}$ & $\begin{array}{c}\text { Pred. } \mathbf{\Delta G} \\
(\mathbf{k J} / \mathbf{m o l})\end{array}$ & $\begin{array}{c}\text { Obs. } \mathbf{\Delta G} \\
(\mathbf{k J} / \mathbf{m o l})\end{array}$ \\
\hline TAR & 52 & 57 & 153 & $172 \pm 9$ \\
\hline TARdb & 49 & 57 & 176 & $183 \pm 10$ \\
\hline 30AU & 64 & 0 & 134 & $147 \pm 8$ \\
\hline $\begin{array}{c}\text { AUGC } \\
\text { (low) }\end{array}$ & 60 & 0 & 160 & $167 \pm 9$ \\
\hline $\begin{array}{c}\text { AUGC } \\
\text { (high) }\end{array}$ & 64 & 100 & 371 & $402 \pm 21$ \\
\hline GCAU & 124 & 50 & 534 & $558 \pm 31$ \\
\hline
\end{tabular}

